\title{
Magnitude Finger Forces Analysis During Simulating Pseudo-Haptic Spring
}

\author{
Jingtao Chen ${ }^{1}$, Peter Mitrouchev ${ }^{2(\otimes)}$, Sabine Coquillart ${ }^{3}$, and Franck Quaine ${ }^{4}$ \\ ${ }^{1}$ Health Simulation Center SimUSanté ${ }^{\circledR}$, Amiens University Hospital, Amiens, France \\ 2 Univ. Grenoble Alpes, CNRS, Grenoble, INP, G-SCOP, 38000 Grenoble, France \\ Peter.Mitrouchev@grenoble-inp. fr \\ 3 Univ. Grenoble Alpes, INRIA, CNRS, Grenoble INP, LIG, 38000 Grenoble, France \\ ${ }^{4}$ Univ. Grenoble Alpes, CNRS, Grenoble INP, GIPSA-Lab, 38000 Grenoble, France
}

\begin{abstract}
This paper focuses on finger force magnitude analysis during stiffness discrimination task. In the frame of their Study and research work MS students from the Université Grenoble Alpes specially designed an experimental bench allowing to simulate a pseudo-haptic spring. Then, a series of stiffness discrimination tests between reals springs and a pseudo-haptic spring were performed. Finger pressing forces and students' (subjects') perception of spring stiffness were recorded and analyzed. The analysis of psychometric curves indicates that subjects underestimate the simulated stiffness of the pseudo-haptic spring. The results also indicate that the peak of finger force applied on pseudo-haptic spring increases as the simulated stiffness increases. Moreover, it was found that the relationships between the logarithm of stiffness and the finger force were linear for the real springs and the pseudo-haptic spring. Pseudo-haptics effect being provided by specially designed isometric force feedback device, the results of this study may be useful for computer-based rehabilitation tasks designed for motor disorder patients with muscle deficiency associated with limited joint movement range or for injured athletes in the process of rehabilitation.
\end{abstract}

Keywords: Muscle force $\cdot$ Pseudo-haptic feedback $\cdot$ Stiffness discrimination

\section{Introduction}

In some simulation such as: medical, sportive, remote surgery..., it is necessary to have stiffness information as it provides useful feedback to discriminate the stiffness, or the compliance, properties of different tissues. Pseudo-haptics is a technique that can provide the illusion of different physical properties such as stiffness, for instance. According to Lécuyer et al. [1] pseudo-haptics is defined as "the generation, augmentation or deformation of haptic sensations by information coming from other sensory modalities", hereinafter referred to as "disruptive modalities".

Besides studies about the influence of user's perception of stiffness, some studies have investigated the hand motor strategy, such as adjustment of peak force intensity or pressing duration, while pressing a real and a pseudo-haptic spring during stiffness 
discrimination tasks. Tan et al. [2] found that higher force is needed for achieving the same magnitude of work cues for less compliant objects during discrimination compared to high compliant objects. The highest peak force is applied to the stiffest objects. This conclusion has been verified by series of studies, no matter whether participants used pinch or press movement [3] to discriminate the stiffness of a real spring.

Endo [4] investigated the exploration of movement, applied force and displacement during stiffness discrimination task with real springs. He has shown that the forces applied on the explored specimen increased with the stiffness until it reached a stabilization for the highest stiffness values. Paljic et al. [5] used pseudo-haptic feedback to simulate a pseudo-haptic torsion spring. They reported that the torque applied on the spring did not vary with its simulated stiffness. However, the comparison between the torques applied on real and pseudo-haptic torsion spring was not addressed. Freyberger et al. [6] investigated the relationship between granularity perception and vibro-tactile coding during stiffness discrimination task.

In [7] Kaim and Drewing investigated to what extent people strategically execute movements that are tuned for softness discrimination of objects with deformable surfaces also during stiffness discrimination tasks. However, a question remains: what is the forcestiffness relationship when pressing a pseudo-haptic spring compared to a real one in stiffness discrimination tasks? The answer to this question may provide a reference model for designing computer-based hand rehabilitation applications, using pseudohaptic feedback. As far as we know, there is no work focusing on this issue.

In this context, the aim of the paper is to investigate the influence of pseudo-haptic feedback on users' finger force. For this purpose, an experimental bench was specifically designed allowing to simulate the pseudo-haptic spring. Its stiffness varies over eleven different percentages, called stiffness percentages $(-40 \%,-30 \%,-20 \%,-10 \%, 0 \%$, $+10 \%,+20 \%,+30 \%,+40 \%,+50 \%$ and $+60 \%$ ) from the targeted stiffness of the compared real spring. After testing both springs, participants had to identify which was stiffer: the real spring or the pseudo-haptic one. Thus, the forces applied on the real springs and pseudo-haptic spring were recorded and analyzed for investigating the trend of change in finger force depending on the change of stiffness of the springs.

\section{Experimental Protocol and Participants}

The experimental protocol presented in [8], where only the displacement of the pseudohaptic spring was visually displayed on a computer screen, was replicated in the performed experiments. Here, the visual stimuli of the two compared springs (real and pseudo-haptic) and their respective displacements were displayed for the subjects on a computer screen for each trial. The experiment includes two parts: $i$ ). Maximal voluntary contraction (MVC) for force and electromyography (EMG) data calibration, ii). Stiffness discrimination tests. MVC consists of three items: $i$ ). Maximal flexion force, $i i)$. Maximal extension force, iii). Maximal co-contraction. Each item is performed consecutively three times and consists in: performing the co-contraction during $3 \mathrm{~s}$. followed by $5 \mathrm{~s}$. break. After each item, subject had $5 \mathrm{~min}$ to rest before starting the stiffness discrimination task. For the maximal flexion and extension forces, subject had to continuously apply the maximum force by vertically pulling and pushing a ring attached 
with a force sensor towards down and towards up. The maximal co-contraction consists in keeping the palm and forearm horizontal, and co-contracting all the forearm muscles. During performing each item, the forces and EMG signal are recorded. During the tests, subjects, in sitting position, had to use the index finger of their dominant hands while applying the forces.

Figure 1 partially shows the test bench for the experiments carried out. Inside a box (Fig. 1a), there are four springs, labeled 1,2, 3 and 4 with physical stiffness of $202 \mathrm{~N} / \mathrm{m}$, $304 \mathrm{~N} / \mathrm{m}, 608 \mathrm{~N} / \mathrm{m}$ and $2500 \mathrm{~N} / \mathrm{m}$ respectively. The real spring is one among springs 1 , 2 and 3 . The pseudo-haptic spring is the stiffest one, namely spring 4 . It has been shown [9], and our own experience confirms, that pseudo-haptic spring simulation is more relevant when using stiffer pseudo-haptic spring. Using stiffness in a similar range for pseudo-haptic and real springs precludes stiffness discrimination task, probably because subjects do not understand the task they must perform. Each spring is situated in a metallic tube and is loaded by the finger force via a button. Beneath each spring, a force sensor (KISTLER 9017B) records the force with a $2000 \mathrm{~Hz}$ sampling frequency during each trial.

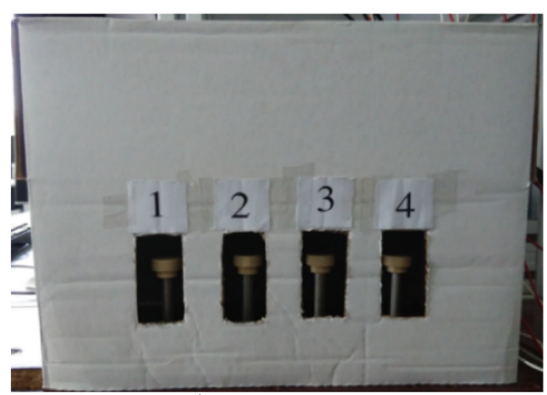

a)

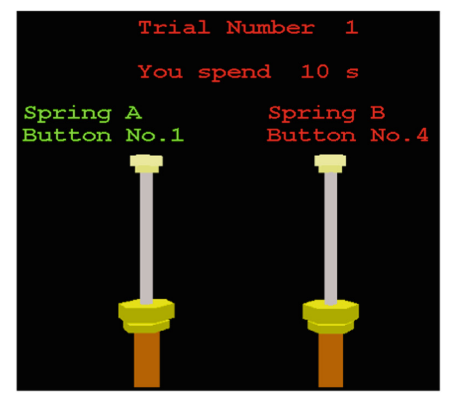

b)

Fig. 1. Experimental setup: (a). Four springs inside the box; (b). a real spring (spring A, left) and the pseudo-haptic spring (spring B, right).

For the real springs no mismatch existed between the visual feedback and its displacement. However, for the pseudo-haptic one a difference existed according to the simulated targeted stiffness value, in order to lure the participants. Previous tests had shown that below $-40 \%$ and above $+60 \%$ the subjects' perception results were no longer influenced by the visual feedback. Thus, the experiments were performed by varying the stiffness of the pseudo-haptic spring randomly over eleven different stiffness percentages from the stiffness of the compared real spring.

During each trial of stiffness discrimination, one of the three real springs was randomly compared to the pseudo-haptic spring whose simulated stiffness varied, as previously said, from the stiffness of the compared real spring by eleven different percentages. The button's displacements of the two compared springs were displayed on the screen labeled "Spring A" and "Spring B". For each spring, the spring number (1 to 4) of the corresponding button was indicated just below "Spring A/B" (Fig. 1b). Subjects started 
a trial by pressing spring $B$ first (i.e. pseudo-haptic spring) and then spring $A$, the randomized compared real springs. After they could switch back and forth between the two springs as they wished. For subject's information, in the top part of the screen, the trials' number and the time spent for this trial were displayed (Fig. 1b). Subjects had to focus on the computer screen and were not allowed to observe the current movements of their fingers while pressing the springs. For each trial, after exploring the springs, they had to press the $S$ button on the keyboard, with their non-dominant hand, to Stop the trial. Then the question: Which spring is stiffer? was displayed on the computer screen. The subjects pushed key $A$ or $B$ to answer the question. The answer was then automatically recorded for further analysis. Fifteen unpaid voluntary and healthy subjects (twelve males and three females), aged from 23 to 25 participated in the experiments. All of them reported no: $i$ ). Visual impairment; ii). Impairments of haptic sensitivity; iii). Diseases or symptoms inducing hand movement disorder.

\section{Results and Discussion}

Figure 2 presents the comparison between the force on real springs and the virtual spring under identical stiffness ( $0 \%$ of change). A significant effect was noted for the spring type $(F=118.32, p$-value $<0.0001)$ and for the stiffness $(F=94.034, p$-value $<0.0001)$ meaning that the force applied on the real springs are lower than this applied on the virtual one, while the force increases from low to higher stiffness values. A significant interaction between the spring type and 'stiffness scale' $(F=8.907, p$-value $<0.001)$ was also observed. The forces applied on real springs increase from $5.946 \mathrm{~N}$ to $11.6 \mathrm{~N}$ and from $9.5 \mathrm{~N}$ to $12.79 \mathrm{~N}$ on virtual spring. This means that the forces applied on the real springs were statistically lower than those applied on the virtual spring when the stiffness of real springs and the virtual spring are the same.

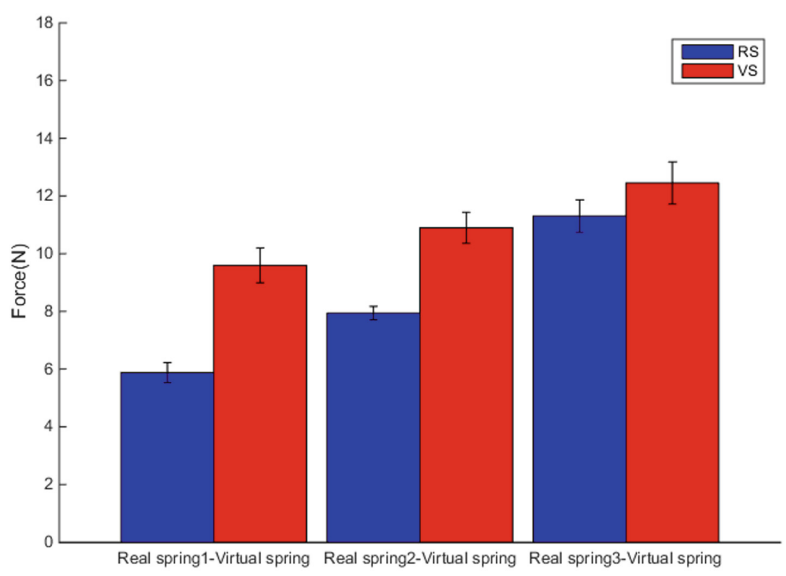

Fig. 2. All paired comparisons have significant differences between force on virtual spring and real springs $(p$-value $<0.0001)$. 
The significant effect of stiffness scale on the force applied on the real springs confirms that higher stiffness induces higher force. This observation also is in agreement with the conclusions in [4]. As no significant effect of stiffness percentage on the force applied on the real springs was found, it can be concluded that the forces applied on the real springs were not significantly influenced by the simulated change in stiffness of the compared pseudo-haptic spring. Since the force-stiffness relationship for both, real and pseudo-haptic spring, fits the logarithmic function, it can be observed: $i$ ). That subject's behavior when pressing the pseudo-haptic spring has the same pattern as when using real one, but with a shift; ii). a similarity is observed between the tendency in the change of force applied on real springs and pseudo-haptic spring when stiffness increases. It appears that for the same stiffness, the force applied on the pseudo-haptic spring is higher than that applied on the real springs. This phenomenon is natural as the physical stiffness of the pseudo-haptic spring is much larger than the stiffness of the real springs. Regardless of the nature of the spring, real or pseudo-haptic, the force and the gap between them (Fig. 2) decreases with the increase in stiffness. In other words, if the simulated stiffness of the pseudo-haptic feedback is higher, less difference exists in the force's behavior pattern between the real springs and the pseudo-haptic spring. That explains the conclusion reported in [4]: the forces reach the same magnitude when the stiffness increases.

\section{Conclusion}

The aim of this study was to investigate the relationship between subjects' finger force applied on springs and spring stiffness through experimental stiffness discrimination tests (comparing the stiffness of real and pseudo-haptic spring). Subjects' perception of spring stiffness and index finger pressing force on the real springs and the pseudo-haptic spring was recorded and analyzed.

Analysis of stiffness discrimination results shows that subjects have underestimated the simulated stiffness of the pseudo-haptic spring as reported in (8). Their perception underestimation on simulated stiffness of pseudo-haptic spring implies that pseudohaptic feedback should be better employed to simulate spring stiffness or biological tissues with higher levels of stiffness.

These findings may be useful in the design and the development of user-centered computer-based rehabilitation applications in general, and for patients with hand motor disorder (limited finger or hand movement ranges) in particular as the isometric-device simulating the pseudo-haptic springs need no displacement.

Acknowledgements. This work was supported by LabEx PERSYVAL-Lab (ANR-11-LABX0025) (https://www.persyval-lab.org/index.html). We would like to thank Mr. Charles-Henry Dufetel and the other MS students for their assistance in the development of the test bench and participation in the experiments. 


\section{References}

1. Lécuyer, A., Burkhardt, J.M., Coquillart, S., Coiffet, P.: "Boundary of illusion": an experiment of sensory integration with a pseudo-haptic system. Proc. IEEE Virtual Reality 2001, 115-122 (2001). https://doi.org/10.1109/vr.2001.913777

2. Tan, H.Z., Durlach, N.I., Beauregard, G.L., Srinivasan, M.A.: Manual discrimination of compliance using active pinch grasp: the roles of force and work cues. Percept. Psychophys. 57(4), 495-510 (1995). https://doi.org/10.3758/bf03213075

3. Freyberger, F.K.B., Färber, B.: Compliance discrimination of deformable objects by squeezing with one and two fingers. In: Proceedings of EuroHaptics, pp. 271-276 (2006a)

4. Endo, H.: Pressing movements and perceived force and displacement are influenced by object stiffness. Physiol. Behav. 163, 203-210 (2016)

5. Paljic, A., Burkhardtt, J.-M., Coquillart, S.: Evaluation of pseudo-haptic feedback for simulating torque: a comparison between isometric and elastic input devices. In: 12th International Symposium on Haptic Interfaces for Virtual Environment and Teleoperator Systems, Proceedings, HAPTICS 2004, pp. 216-223 (2004). https://doi.org/10.1109/haptic.2004.128 7199

6. Freyberger, F.K.B., Farber, B.: Psychophysics and perceiving granularity. In: 2006 14th Symposium on Haptic Interfaces for Virtual Environment and Teleoperator Systems, pp. 387-393 (2006b). https://doi.org/10.1109/HAPTIC.2006.1627105

7. Kaim, L., Drewing, K.: Exploratory strategies in haptic softness discrimination are tuned to achieve high levels of task performance. IEEE Trans. Haptics 4(4), 242-252 (2011). https:// doi.org/10.1109/toh.2011.19

8. Lécuyer, A., Coquillart, S., Kheddar, A., Richard, P., Coiffet, P.: Pseudo-haptic feedback: can isometric input devices simulate force feedback? In: Feiner, S., Thalmann, D. (eds.) Proceedings IEEE Virtual Reality (2000). https://doi.org/10.1109/vr.2000.840369

9. Wu, W., Basdogan, C., Srinivasan, M.A., Basdogan, C.: Visual, haptic, and bimodal perception of size and stiffness in virtual environments. In: Proceeding of the ASME Dynamic Systems and Control Division Bd, p. 67 (1999)

Open Access This chapter is licensed under the terms of the Creative Commons Attribution 4.0 International License (http://creativecommons.org/licenses/by/4.0/), which permits use, sharing, adaptation, distribution and reproduction in any medium or format, as long as you give appropriate credit to the original author(s) and the source, provide a link to the Creative Commons license and indicate if changes were made.

The images or other third party material in this chapter are included in the chapter's Creative Commons license, unless indicated otherwise in a credit line to the material. If material is not included in the chapter's Creative Commons license and your intended use is not permitted by statutory regulation or exceeds the permitted use, you will need to obtain permission directly from the copyright holder.

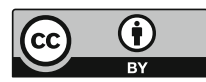

